\title{
Experimental random-party entanglement distillation via weak measurement
}

\author{
Zheng-Da Li, ${ }^{1,2,3,{ }^{*}}$ Xiao Yuan, $, 1,2,3,{ }^{*}$ Xu-Fei Yin,,${ }^{1,2,3,{ }^{*}}$ Li-Zheng Liu, ${ }^{1,2,3}$ Rui Zhang,,${ }^{1,2,3}$ Yue-Yang Fei, ${ }^{1,2,3}$ Li Li,,${ }^{1,2,3}$ \\ Nai-Le Liu, ${ }^{1,2,3}$ Xiongfeng Ma, ${ }^{4} \mathrm{He} \mathrm{Lu},{ }^{5}$ Yu-Ao Chen $\odot,{ }^{1,2,3}$ and Jian-Wei Pan ${ }^{1,2,3}$ \\ ${ }^{1}$ Hefei National Laboratory for Physical Sciences at Microscale and Department of Modern Physics, \\ University of Science and Technology of China, Hefei 230026 China \\ ${ }^{2}$ Shanghai Branch, CAS Center for Excellence and Synergetic Innovation Center in Quantum Information and Quantum Physics, \\ University of Science and Technology of China, Shanghai 201315 China \\ ${ }^{3}$ Shanghai Research Center for Quantum Sciences, Shanghai 201315, China \\ ${ }^{4}$ Center for Quantum Information, Institute for Interdisciplinary Information Sciences, Tsinghua University, Beijing 100084, China \\ ${ }^{5}$ School of Physics, Shandong University, Jinan 250100, China
}

(Received 1 April 2019; revised manuscript received 23 January 2020; accepted 20 March 2020; published 16 April 2020)

\begin{abstract}
Maximally bipartite entangled state $\left|\Psi^{+}\right\rangle$, also known as the Einstein-Podolsky-Rosen pair, is the unit resource of entanglement and the key for quantum information processing. An important problem is that how many maximally bipartite entangled states could be distilled from a multipartite entangled state shared among a quantum network. Here, we focus on the distillation of $\left|\Psi^{+}\right\rangle$from a single copy of the three-qubit $W$ state. An interesting phenomenon in this case is that the random entanglement distillation between two unspecified parties can yield a strictly higher distillation rate (the average number of $\left|\Psi^{+}\right\rangle$distilled from each $W$ state) than the case between two specified parties. In this work, we develop a distillation protocol by introducing weak measurements that do not destroy the global entanglement. We find that the distillation rate can be significantly enhanced with only a few rounds by performing an extra distillation procedure between two specified parties at the final step. Experimentally, we prepare a three-photon $W$ state in the polarization degree of freedom, and employ the path degree of freedom of each photon as probe qubits to realize the weak measurement. As a proof-of-principle demonstration, we show that the distillation rate is enhanced from $2 / 3$, which is the theoretical limit of any distillation scheme between two specified parties, to $0.751 \pm 0.030$ between two unspecified parties with only one distillation round.
\end{abstract}

DOI: 10.1103/PhysRevResearch.2.023047

\section{INTRODUCTION}

Multipartite entanglement is an essential resource in a quantum network that benefits quantum key distribution [1], quantum teleportation [2-4], and distributed quantum computation. When a network with one class of multipartite entanglement is established, it can be hard to convert it to another class of multipartite entanglement via local operations and classical communication (LOCC). For instance, in the tripartite scenario, a single copy of Greenberger-Horne-Zeilinger (GHZ) state cannot be converted to a W-like state via any LOCC even probabilistically [5]. Starting with the maximally entangled bipartite state, known as the Einstein-PodolskyRosen (EPR) pair, it is flexible to generate different classes of multipartite entangled states [6]. Therefore it is convenient to consider EPR pairs as the fundamental building block for multipartite entanglement preparation.

${ }^{*}$ These authors contributed equally to this work.

Published by the American Physical Society under the terms of the Creative Commons Attribution 4.0 International license. Further distribution of this work must maintain attribution to the author(s) and the published article's title, journal citation, and DOI.
In practice, it is natural to consider the question: after some quantum information processing (QIP) tasks, where byproducts of multipartite entangled states shared among the network are created, how could one reshape them into standard EPR pairs? A trivial solution is to discard the multipartite entanglement and re-distribute EPR pairs. However, in some quantum network scenarios, especially when the geometry of network cannot be changed, it may be inconvenient or costly to distribute EPR pairs between two desired nodes. On the other hand, from the resource perspective, this is not the optimal way and a more economic strategy is to recycle the entanglement. Therefore entanglement distillation from multipartite entangled states is of great importance in the future quantum internet [7].

Here we investigate the distillation of EPR pairs from a specific multipartite entangled state-the $W$ state, which has wide applications in QIP tasks such as leader election in anonymous network [9] and asymmetric telecloning [10]. Considering a three-qubit $W$ state $\left|W_{3}\right\rangle$ shared by three parties Alice, Bob, and Charlie

$$
\left|W_{3}\right\rangle=\frac{1}{\sqrt{3}}(|001\rangle+|010\rangle+|100\rangle)_{A B C},
$$

a naive strategy to distill EPR pairs is to perform a strong $Z$ basis measurement on the third party as shown in Fig. 1(a). It 
(a)

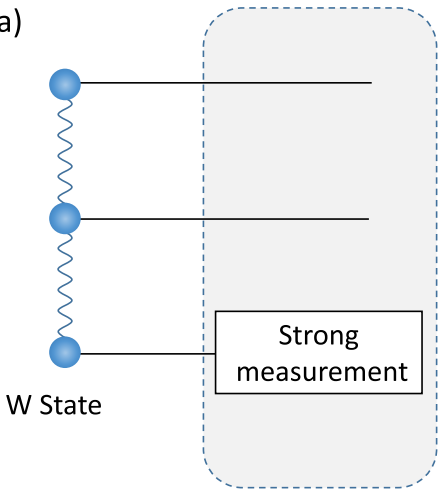

(c)

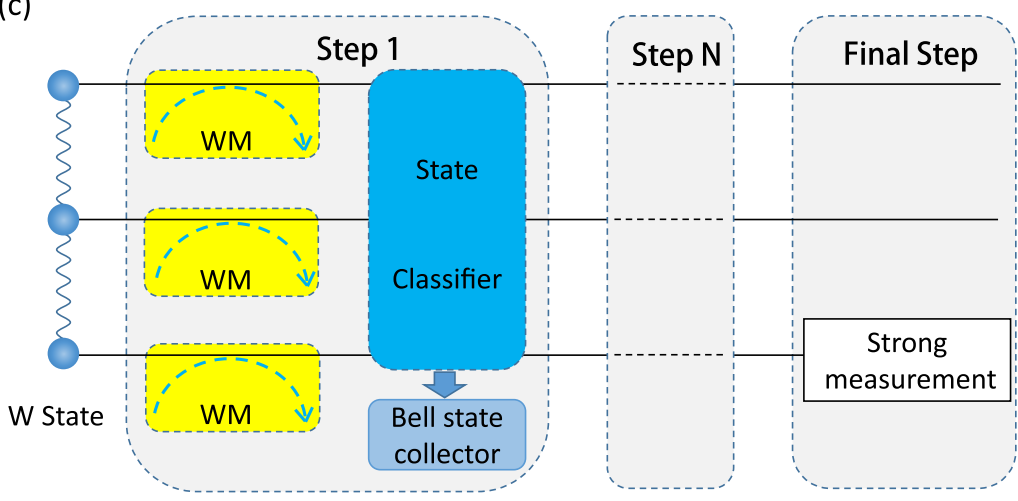

(b)

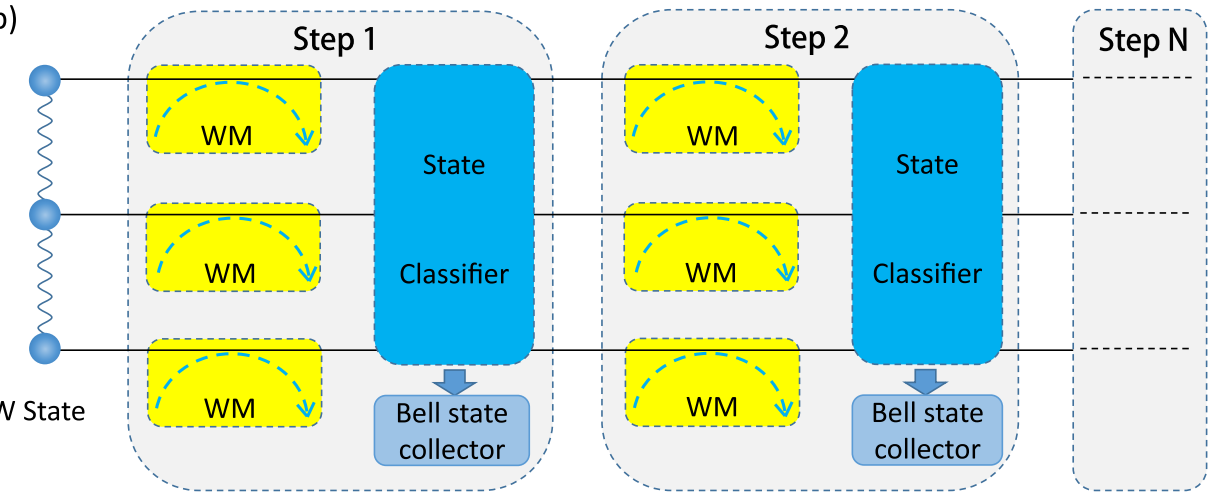

(d)

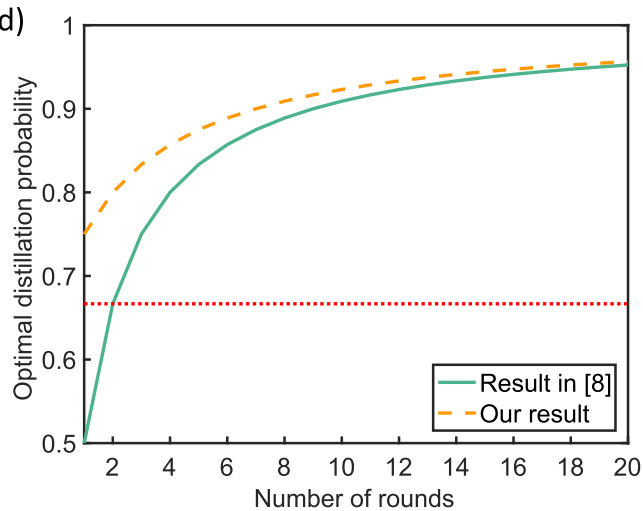

FIG. 1. Schematic diagram of distillation scheme for a tripartite $W$ state. (a) The optimal specified distillation scheme for the $W$ state with a single copy. (b) The original random distillation scheme proposed by Fortescue and Lo. (c) The modified scheme with an extra specified distillation procedure at the final step. (d) Comparison of the optimal distillation rate between the modified and original schemes. The solid line denotes the result in Ref [8], the dash line denotes our result, and the dot line represents the bound of distillation between two specified parties.

destroys the correlation between the first two and the third party, and preserves the correlation between the first two parties. On average, one can obtain $2 / 3$ EPR pairs from a single copy of $\left|W_{3}\right\rangle$ between any two specified parties such as Alice and Bob with this strategy, which is shown to be optimal via strong measurements [11]. However, it is notable that the entanglement of the $W$ state exist globally on all the three parties and such a distillation strategy does not exploit the merit of the global entanglement. In general, the distilled EPR pair could exist on any two of the three parties. Thus the global entanglement will lead to a distilled state that is in a superposition of the three cases, that is, EPR pair between Alice and Bob, Bob and Charlie, and Charlie and Alice. If we only focus on the average number of EPR pairs distilled, this is equivalent to the case of entanglement distillation between two unspecified parties, named random distillation $[8,12,13]$. Surprisingly, without specifying the two parties that the EPR pair is distilled to, it is possible to achieve a higher distillation rate for $W$-like states. We also note that such an interesting phenomenon does not occur on GHZ-like states.

In the seminal work by Fortescue and Lo [8], they proposed a scheme to randomly distill one EPR pair from one $W$ state by performing an infinite round of weak measurements [14]. Each operation is only weakly performed, so that it allows one to distill the EPR pair without completely destroying the global entanglement. The more rounds of the weak measurements are performed, the higher distillation rate can be obtained. However, there are two technical obstacles in realizing Fortescue and Lo's scheme, one is that it is unclear about how to experimentally implement the weak measurements and another one is that it requires at least three rounds of weak measurements to beat the $2 / 3$ bound of distillation between two specified parties.

In this paper, we theoretically optimize the original random distillation scheme which significantly improves the distillation rate especially when the number of rounds is small. In experiment, we encode the $W$ state in the polarization degree of freedom of photons and realize the weak measurement with the path degree of freedom. As a proof-of-principle demonstration of our scheme, we experimentally show that the rate of distilling EPR pair from one three-photon $W$ state is enhanced to $0.751 \pm 0.030$ by only performing one round of weak measurement.

\section{THEORY}

Let us start by revisiting the original $W$ state random distillation scheme in Ref. [8] as shown in Fig. 1(b). Suppose Alice, Bob, and Charlie share a three-qubit $W$ state in Eq. (1), and they attempt to distill an EPR pair on any two of the three parties via LOCC. Then, three probe qubits are involved to couple with the system qubits to perform weak measurement and the positive-operator-valued measure (POVM) elements 

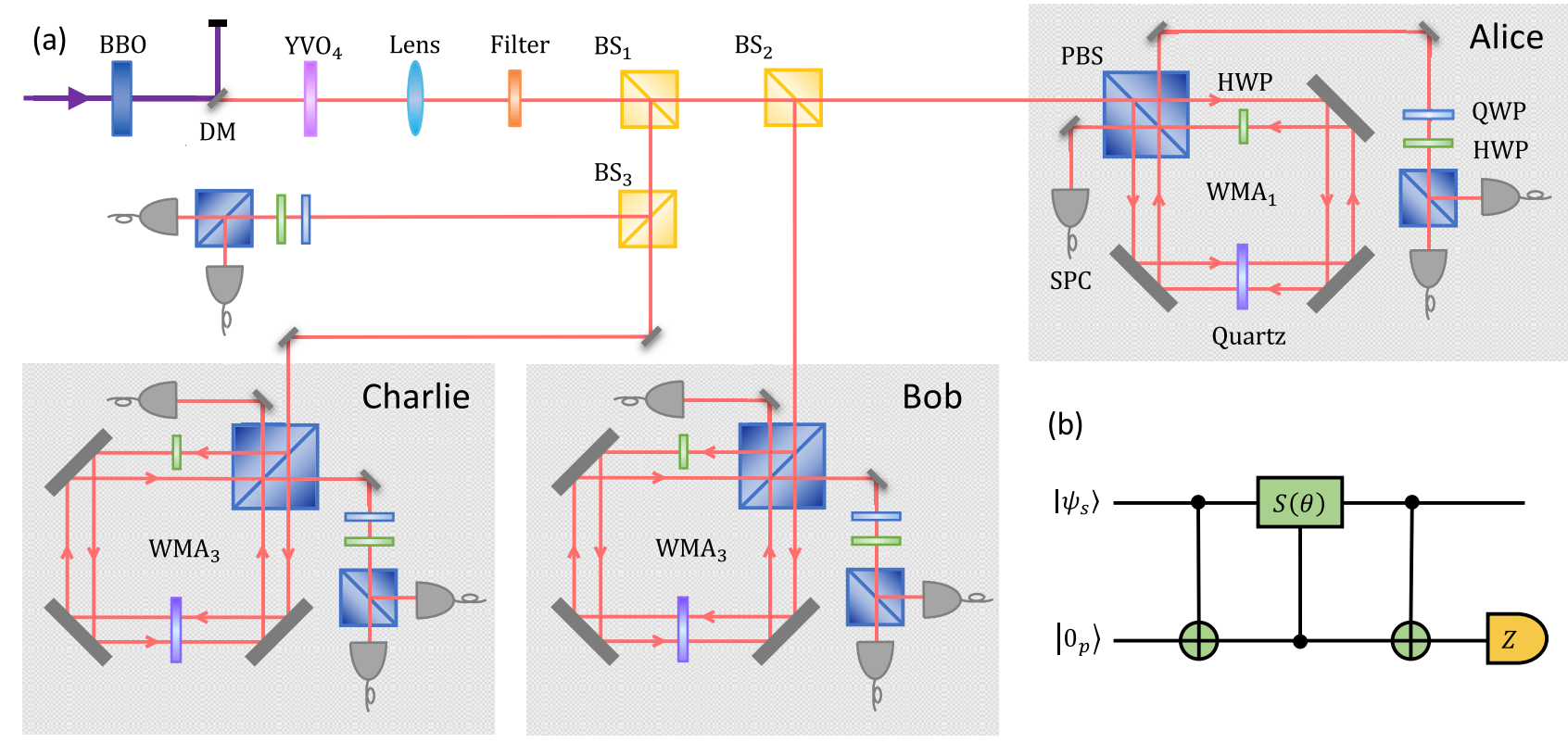

FIG. 2. Schematic of the experimental setup. (a) An ultraviolet laser (150 fs, $76 \mathrm{MHz}, 300 \mathrm{~mW})$ is focused on a 2-mm-thick collinear $\beta$-barium borate (BBO) crystal to generate down-converted photons. A 0.715 -mm-thick Yttrium orthovanadate $\left(\mathrm{YVO}_{4}\right)$ crystal is used to eliminate the spatial and temporal walk-off. Narrow-band filters with a full width at half of the transmittance maximum (FWHM) of $\Delta \lambda=3 \mathrm{~nm}$ are used to eliminate the frequency correlations between down-converted photons. The weak measurement apparatus (WMA) with adjustable measurement strength are implemented by a Sagnac interferometer. The measurement system is consisted of a quarter-wave plate (QWP), a half-wave plate (HWP), a polarizing beam splitter (PBS) and two single-mode fiber coupled single-photon detectors in each output of PBS. (b) A quantum circuit illustration of implementing the weak coupling with a Sagnac interferometer.

should be in the form of

$$
\mathcal{O}_{+}=\left(1-\frac{R}{2}\right) \mathbb{1}+\frac{R}{2} Z, \quad \mathcal{O}_{-}=\frac{R}{2} \mathbb{1}-\frac{R}{2} Z,
$$

where $\mathrm{Z}$ is the Pauli matrix, $R$ is the measurement strength of the weak measurement. By changing the value of $R$ from 0 to 1 , the POVM changes from the identity (no measurement) to a projector (strong measurement).

After Alice, Bob, and Charlie perform weak measurements simultaneously, the measurement results can be divided into three situations. (1) If all the three parties get outcome " $\mathcal{O}_{+}$", with probability $(1-R)^{2}$, they share a $W$ state again and we just repeat the weak measurements. (2) If only one of the three parties gets outcome " $\mathcal{O}_{-}$" with probability $2 R(1-R)$, the other two parties share an EPR pair and the scheme aborts. (3) If two or more parties get outcome " $\mathcal{O}_{-}$" with total probability $R^{2}$, the $W$ state is converted to a product state and the scheme aborts.

Suppose weak measurements are repeated at most $N$ rounds before the scheme aborts, then the optimal distillation rate of obtaining EPR pair from one $|W\rangle$ is

$$
\left\langle E_{N}^{\mathrm{opt}}\right\rangle=\frac{N}{N+1},
$$

with the optimal strength $R_{i}^{\mathrm{opt}}=1 /(N-i+2)$ for the $i$ th round. The distillation probability approaches one when $N$ goes to infinity. For finite number of rounds, the original distillation scheme is not optimal as the $W$ state obtained in situation 1 is not further exploited in the last round.
Here, we propose to perform an additional distillation procedure on this $W$ state by performing a strong measurement to get an EPR on two specified parties with probability $2 / 3$ [Fig. 1(c)]. That is, for this $W$ state, we directly make a strong measurement on Charlie's qubit and we obtain an EPR pair between Alice and Bob with probability $2 / 3$. Therefore, by optimizing the transformation parameter $R$ for each round, we get a better distillation rate

$$
\left\langle E_{N}^{\text {opt,new }}\right\rangle=\frac{N+2}{N+3},
$$

with $R_{i}^{\mathrm{opt}}=1 /(N-i+4)$ for the $i$ th round. Notably, our strategy of exploiting the $W$ state in the last round significantly improves the performance especially with a small number of rounds as shown in Fig. 1(d). This is especially meaningful in practice as it is experimentally challenging to realize a large number of rounds of weak measurements. Interestingly, to beat the distillation rate $2 / 3$ on two specified parties with operations acting on a single copy of $W$ state, our modified scheme only needs at least a single round. In the following, we present an experimental demonstration of the modified random distillation scheme with a photonic system.

\section{EXPERIMENTAL IMPLEMENTATION}

\section{A. State preparation}

In the experiment, we first generate a three-photon $W$ state, $|W\rangle=1 / \sqrt{3}(|H V V\rangle+|V H V\rangle+|V V H\rangle)$, in the polarization degree of freedom [15], where $H$ and $V$ represent the horizontal and vertical polarization, respectively. The setup 


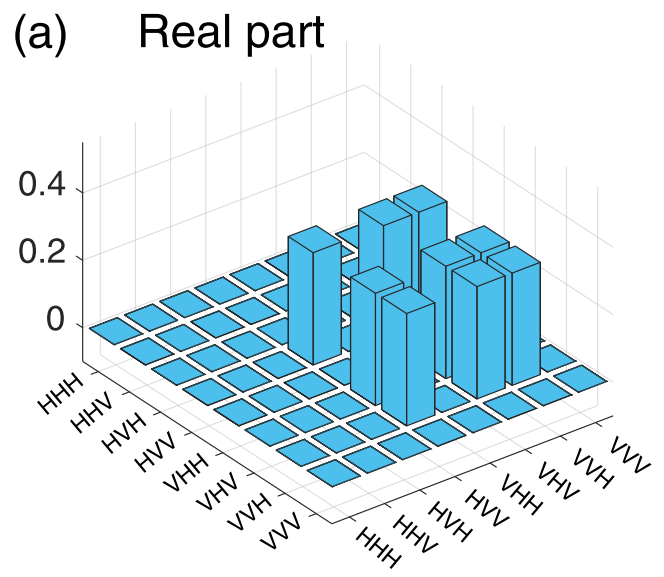

\section{Imaginary part}

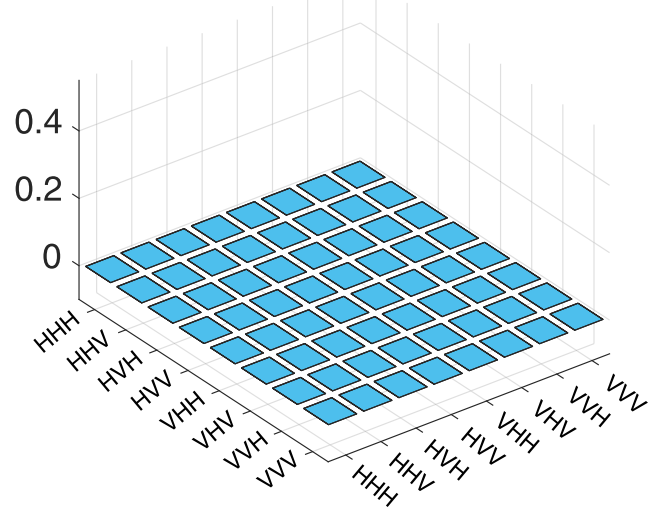

\section{Imaginary part}
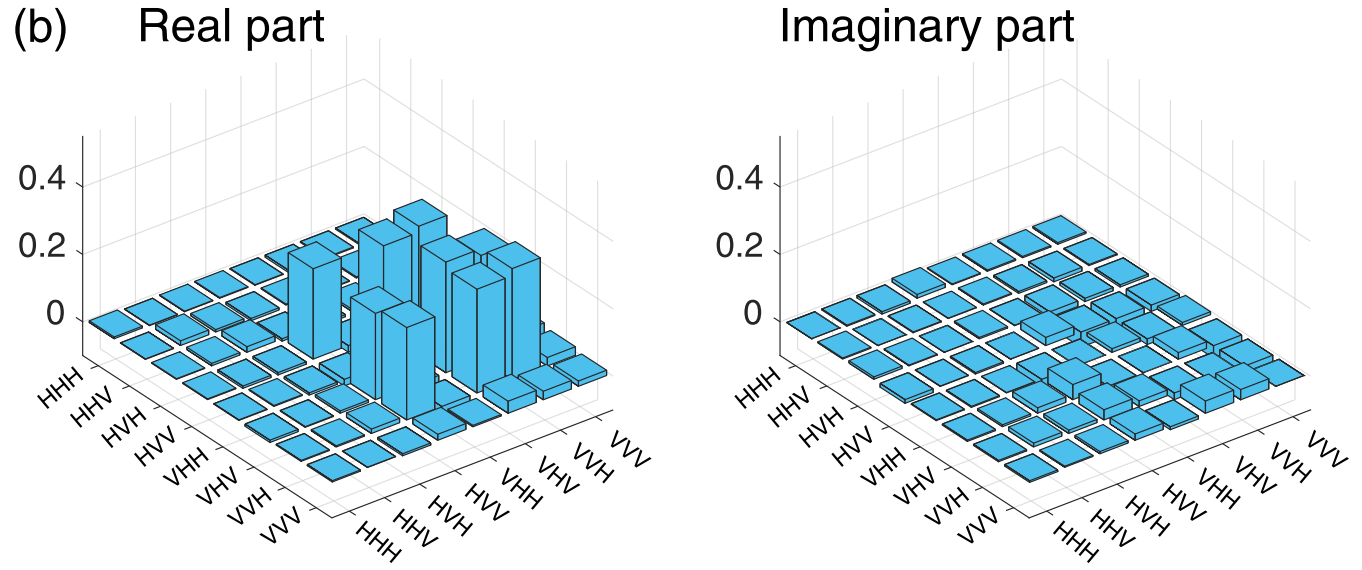

FIG. 3. (a) The density matrix of the ideal $|W\rangle$. (b) The experimentally reconstructed density matrix $\rho_{W}^{\exp }$. The fidelity with the reconstructed density matrix $\rho_{W}^{\exp }$ is $F=\operatorname{Tr}\left(\rho_{W}^{\exp }|W\rangle\langle W|\right)=0.866 \pm 0.007$.

to generate $|W\rangle$ is illustrated in Fig. 2(a). An ultraviolet pulse $(390 \mathrm{~nm}, 130 \mathrm{fs}, 76 \mathrm{MHz}$ ) passes through a 2-mmthick collinear $\beta$-barium borate (BBO) crystal. The second order of spontaneous parametric down conversion (SPDC) process in $\mathrm{BBO}$ contributes $\left|H_{A} V_{A} V_{B} H_{B}\right\rangle$, which is utilized to prepare $|W\rangle$. As shown in Fig. 2(a), the four photons are probabilistically distributed among the four spatial modes by three beam splitters (BSs). Once we detect one photon in each mode, we postselectively observe a four-photon Dick state

$$
\begin{aligned}
\left|D_{4}\right\rangle= & \frac{1}{\sqrt{6}}(|H H V V\rangle+|H V H V\rangle+|H V V H\rangle \\
& +|V H H V\rangle+|V H V H\rangle+|V V H H\rangle) .
\end{aligned}
$$

Then, we trigger the photon in the fourth mode into state $|H\rangle$ and obtain the target state $|W\rangle=\frac{1}{\sqrt{3}}(|V V H\rangle+|V H V\rangle+$ $|H V V\rangle)_{A B C}$ in the other three modes. We perform the full tomographic measurements on the experimentally generated $\rho_{W}$ state (Fig. 3) and calculate the fidelity $\operatorname{Tr}\left(|W\rangle\langle W| \rho_{W}\right)=$ $0.866 \pm 0.007$ [16]. (See Appendix A for more details.)

\section{B. Distillation}

Recall the distillation procedure, the key step is performing the weak measurement [as shown in Fig. 1(c)]. In our experiment, we design a weak measurement apparatus (WMA) with adjustable measurement strength utilizing a Sagnac interferometer as shown in Fig. 2(a). In the WMA, the probe qubit is encoded with the path degree of freedom of photons. And $\left|0_{p}\right\rangle\left(\left|1_{p}\right\rangle\right)$ represents the clockwise (counterclockwise) propagation states in the Sagnac interferometer. The Sagnac interferometer could be considered as a 2-qubit unitary gate $\mathrm{U}$ between the system and probe qubit, with $\mathrm{U}=$ C-NOT - C-S $(\theta) \cdot$ C-NOT as shown in Fig. 2(b). Here, C-NOT is the controlled-NOT gate defined as C-NOT $=|H\rangle\langle H| \otimes$ $\mathbb{1}_{p}+|V\rangle\langle V| \otimes X_{p}$ and implemented by a PBS with the system qubit as the control and the probe qubit as the target with $X$ being the Pauli- $X$ matrix. While C- $S(\theta)_{p s}=\mathbb{1} \otimes\left|0_{p}\right\rangle\left\langle 0_{p}\right|+$ $(\cos 2 \theta Z+\sin 2 \theta X) \otimes\left|1_{p}\right\rangle\left\langle 1_{p}\right|$ is the controlled-rotation gate which is implemented by a half-wave plate oriented at $\theta$ with the probe qubit as the control and system qubit as the target. At last, we measure the probe qubit in the Pauli- $Z$ basis. With outcome $z=1(z=-1)$, we perform the measurement $\mathcal{O}_{+}$ $\left(\mathcal{O}_{-}\right)$defined in Eq. (2) on the system qubits

$$
\begin{aligned}
& \mathcal{O}_{+}=\left(1-\frac{\sin ^{2} 2 \theta}{2}\right) \mathbb{1}+\frac{\sin ^{2} 2 \theta}{2} Z, \\
& \mathcal{O}_{-}=\frac{\sin ^{2} 2 \theta}{2} \mathbb{1}-\frac{\sin ^{2} 2 \theta}{2} Z .
\end{aligned}
$$


The corresponding measurement strength of the weak measurement is $R=\sin ^{2} 2 \theta$, and we can continuously change it by adjusting the HWP's angle $\theta$ in the Sagnac interferometer. (See Appendix $\mathrm{C}$ for more details.)

After performing the weak measurements on the three parties, three outcomes can be observed in our experiment. The outcome $\mathcal{S}_{1}$ means no photon clicked on the detectors of the three WMAs. According to situation 1 of the distillation procedure, the remainder state is still a $|W\rangle$ with an ideal probability $P_{\mathcal{S}_{1}}^{\text {ideal }}=\left(1-R^{2}\right)$. The outcome $\mathcal{S}_{2}$ indicates that one and only one detector of WMA clicks. According to situation 2 , we will have $\left|\Psi^{+}\right\rangle=\frac{1}{\sqrt{2}}(|01\rangle+|10\rangle)$ at any two of $A, B$ and $C$ with an ideal probability of $P_{\mathcal{S}_{2}}^{\text {ideal }}=2 R(1-R)$. The outcome $\mathcal{S}_{3}$ represents two or more photons click on the detectors of three WMAs. According to situation 3, the distillation procedure will fail with an ideal probability of $P_{\mathcal{S}_{3}}^{\text {ideal }}=R^{2}$.

Next, we show that hybridizing with specified distillation scheme, the distillation rate of our one-round distillation scheme can beat $2 / 3$. In situation 1 , when three photons outcome with $\mathcal{S}_{1}$, we do not send them to further distillation. Instead, we distill an EPR pair using specified distillation scheme with the ideal probability $\frac{2}{3} \times P_{\mathcal{S}_{1}}^{\text {ideal }}$. Thus the ideal probability of this distillation scheme in our experiment is calculated as $P^{\text {ideal }}=\frac{2}{3} \times P_{\mathcal{S}_{1}}^{\text {ideal }}+P_{\mathcal{S}_{2}}^{\text {ideal }}=\frac{2}{3}\left(-2 R^{2}+R+1\right)$, where we can obtain a maximum $P^{\text {ideal }}=0.75$ with $R=1 / 4$.

\section{Result}

We firstly measure the probabilities of $P_{\mathcal{S}_{1}}^{\exp }, P_{\mathcal{S}_{2}}^{\exp }, P_{\mathcal{S}_{3}}^{\exp }$, and the probability of specified distillation $P_{\text {specified }}^{\exp }$ under the condition that weak measurement strength $R=1 / 4$. As shown in Fig. 4(a), we observe that $P_{\mathcal{S}_{1}}^{\exp }=0.533 \pm 0.022$, $P_{\mathcal{S}_{2}}^{\exp }=0.416 \pm 0.021, P_{\mathcal{S}_{3}}^{\exp }=0.051 \pm 0.010$, and $P_{\text {specified }}^{\exp }=$ $0.630 \pm 0.029$. Thus we calculate the distillation rate $P^{\exp }=$ $P_{\text {specified }}^{\exp } \times P_{\mathcal{S}_{1}}^{\exp }+P_{\mathcal{S}_{2}}^{\exp }=0.751 \pm 0.030$, which is beyond the bound $2 / 3$ for a specific scheme.

We also measure and calculate the distillation rate $P^{\exp }=$ $P_{\text {specified }}^{\text {exp }} \times P_{\mathcal{S}_{1}}^{\text {exp }}+P_{\mathcal{S}_{2}}^{\text {exp }}$ under different measurement strength $R$. As shown in Fig. 4(b), our experimental results are in good agreement with the theoretical expectations. Furthermore, we measure the fidelity of the distilled $\left|\Psi^{+}\right\rangle$in our experiment and observe an average fidelity of $F=0.873 \pm 0.018$ (see Appendix B for more details). It is worth remarking that the infidelity mainly comes from the imperfect preparation of the input $W$ state. The fidelity of the distilled EPR pair can be significantly improved given an ideal $W$ state or further enhanced by performing a second round of distillation on multiple copies of noisy EPR pairs.

Moreover, the WMA in our experiment could be used to as a key element of the setup to achieve an arbitrary $N$-round entanglement distillation with high-speed feedback operations and high-precision time control (see Appendix D).

\section{CONCLUSION}

It is an interesting open problem to investigate entanglement manipulation of general multipartite entangled states. In this work, we theoretically propose a modified distillation scheme that gives a significantly higher distillation rate than
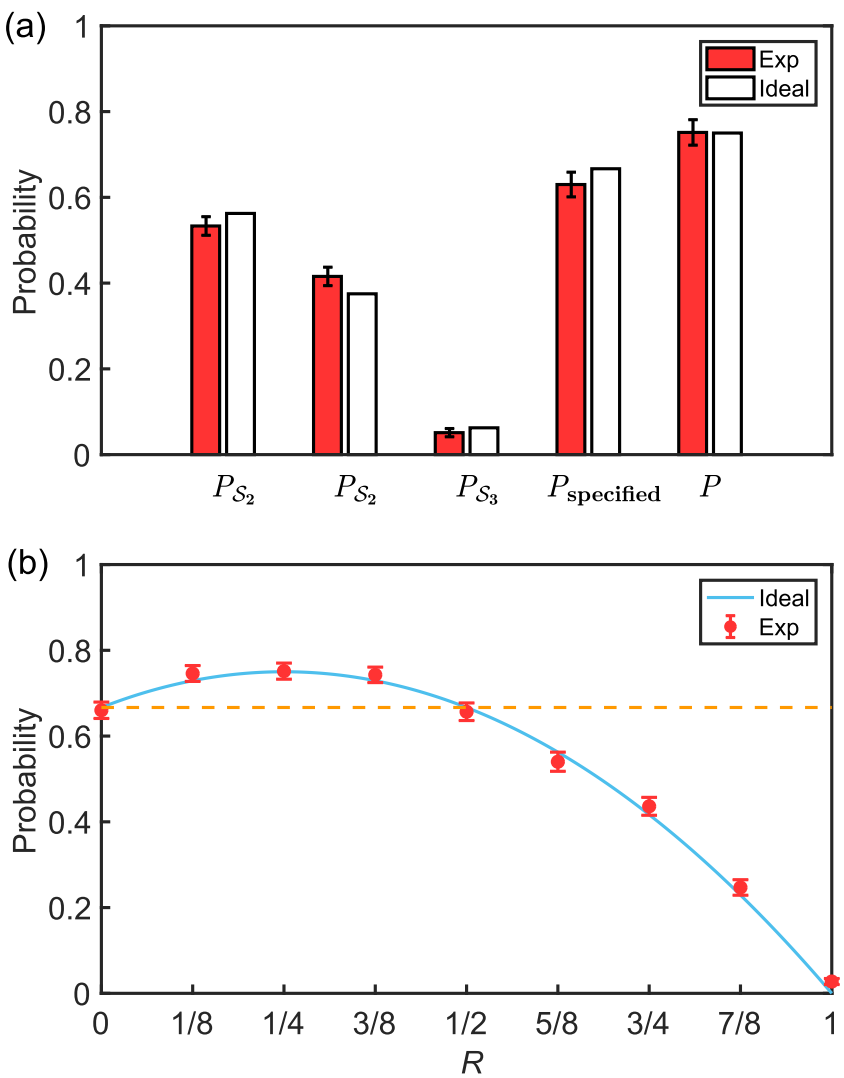

FIG. 4. (a) Measured probabilities of $P_{\mathcal{S}_{1}}^{\exp }, P_{\mathcal{S}_{2}}^{\exp }, P_{\mathcal{S}_{3}}^{\text {exp }}, P_{\text {specified }}^{\text {exp }}$, and $P^{\text {exp }}$. The left red filled histogram represent the experimental results, and the right blank histogram represent the ideal value. The slight mismatch between the ideal value and the experimental results is caused by the imperfections in the prepared $\rho_{W}$. (b) Measured probability of distillation under different measurement strength $R$, the solid line represents the ideal probability of distillation and the dashed line represents the bound of distillation between two specified parties.

the original scheme with a few experiment rounds and experimentally realize it by introducing an auxiliary path degree of freedom. Compared to conventional entanglement distillation schemes that generally require apply joint and deterministic distillation operations on many copies of states $[17,18]$, our work only performs individual operations on a single copy of the tripartite $W$ state. We show that entanglement distillation into unspecified two parties can easily beat the $2 / 3$ bound of distillation between two specified parties. Our results highlight the power of optical realization of random entanglement distillation and quantum networks. Future works can be directed to theoretical studies of general multipartite entangled states and experiments with genuine feed-forward operations.

\section{ACKNOWLEDGMENTS}

We thank Luo-Kan Chen for helpful comments and discussions. This work was supported by the National Natural Science Foundation of China (Grants No. 11975222, No. 61771443, No. U1738140, No. 11875173, No. 11674193, and No. 11974213), the National Key R\&D Program of China (Grants No. 2017YFA0303900, No. 2017YFA0304004, and 
No. 2019YFA0308200), the Anhui Initiative in Quantum Information Technologies and the Chinese Academy of Sciences, the Shanghai Municipal Science and Technology Major Project (Grant No. 2019SHZDZX01).

\section{APPENDIX A: STATE CHARACTERIZATION OF $\rho_{W}$}

We do the full tomographic measurements on the prepared $W$ state to reconstruct the density matrix $\rho_{W}^{\exp }$ [16]. Here, the measurement basis is chosen as $\{|l\rangle|m\rangle|n\rangle\}$ with $|l\rangle,|m\rangle,|n\rangle \in$ $[|H\rangle,|V\rangle,|D\rangle,|A\rangle,|L\rangle,|R\rangle]$. Then, 216 measurement outcomes in total are measured. However, the orthogonal states could be measured simultaneously in the experiment. In practice, only 27 measurement settings are required. For each measurement setting, the fourfold coincidence is accumulated in 30 minutes with coincidence rate $\sim 15 \mathrm{~min}^{-1}$. The results are shown in Fig. 3 in the main text. We calculate the fidelity with the reconstructed density matrix $\rho_{W}$, and obtain the fidelity $F=\operatorname{Tr}\left(\rho_{W}^{\exp }|W\rangle\langle W|\right)=0.866 \pm 0.007$.

We also show that the prepared $W$ state is genuinely tripartite entangled using the following entanglement witness $[19,20]$

$$
\mathcal{W}=\frac{2}{3} \mathbb{I}-|W\rangle\langle W|
$$

By using the reconstructed density matrix $\rho_{W}^{\exp }$, we calculate that $\langle\mathcal{W}\rangle=\operatorname{Tr}\left(\mathcal{W} \rho_{W}^{\exp }\right)=-0.243 \pm 0.014$. Since all separable states has a positive witness measure, it indicates that the prepared $W$ state is a genuinely tripartite entangled state.

\section{APPENDIX B: ADDITIONAL RESULTS OF THE DISTILLATION}

The probability $P_{\mathcal{S}_{2}}^{\exp }$ is the sum of the three probabilities that only one out of three detectors of weak measurement apparatus (WMA) clicks. We show the measured probability of each case in Table I.

In our scheme, $\left|\Psi^{+}\right\rangle$is distilled from unspecified parties and specified parties. For each case, we calculate the fidelity of $\left|\Psi^{+}\right\rangle$by measuring the decomposed local observers $\left|\Psi^{+}\right\rangle\left\langle\Psi^{+}\right|=1 / 2(|H V\rangle\langle H V|+| V H\rangle\langle V H|)+$ $1 / 4(X X+Y Y)$. The results are shown in Fig. 5(a).

If the three outcomes on Alice, Bob, and Charlie are all $\mathcal{O}_{1}$, the state is $|W\rangle$, which is the same as the initial state and can be sent to another distillation round. We measure the fidelity of this remainder $|W\rangle$ by doing the full tomographic measurements. As shown in Fig. 5(b), we calculate its fidelity to be $F=0.863 \pm 0.002$. The result indicates that the state is not affected by the distillation device and it is suitable for further distillation.

TABLE I. The probabilities of three cases in unspecified distillation

\begin{tabular}{lcc}
\hline \hline Parties & Exp & Ideal \\
\hline B and C & $0.157 \pm 0.016$ & 0.125 \\
A and C & $0.131 \pm 0.015$ & 0.125 \\
A and B & $0.127 \pm 0.015$ & 0.125 \\
\hline \hline
\end{tabular}
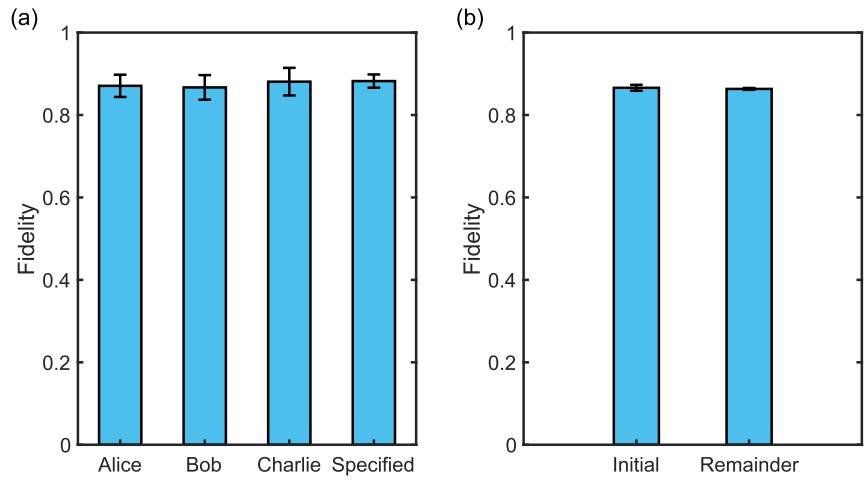

FIG. 5. (a) The fidelity of distilled $\left|\Psi^{+}\right\rangle$. The label Alice, Bob, and Charlie in $x$ axis express the outcome " $z=-1$ " is appeared on Alice, Bob, and Charlie respectively, and the fidelity is $F_{\mathrm{Alice}}=$ $0.870 \pm 0.027, F_{\mathrm{Bob}}=0.867 \pm 0.030$, and $F_{\text {Charlie }}=0.881 \pm 0.034$ Label specified represents $\left|\Psi^{+}\right\rangle$is distilled using specified distillation scheme, and the fidelity is $F_{\text {Specified }}=0.882 \pm 0.016$, (b) The fidelity of the initial and remainder $W$ state.

\section{APPENDIX C: EXPERIMENTAL IMPLEMENTATION OF THE WEAK MEASUREMENT}

Weak measurement provides an effective way to gain little information about a quantum system without completely destroy it [14], which has been widely used in signal amplification, state tomography and the test of error-disturbance uncertainty relations [21-23]. In this work, we apply the weak measurement method into random entanglement distillation to achieve a higher distillation rate for a $W$ state [8].

Then, how to design a weak measurement is an important problem in our work. In the experiment, we should implement the weak measurement for a system qubit encoded with polarization degree of freedom of photons, with $|H\rangle$ and $|V\rangle$ represent the horizontal and vertical polarization, respectively. To achieve the weak measurement, the path degree of freedom of photons is inserted as the probe qubit $(p)$ shown in Fig. 2(b) in the main text. Then the photon is guided into a Sagnac interferometer as shown in Fig. 2(a) in the main text. We let $\left|0_{p}\right\rangle$ represent the clockwise propagation states in the Sagnac interferometer, and $\left|1_{p}\right\rangle$ represent the counterclockwise propagation states. The Sagnac interferometer could be considered as a 2-qubit unitary gate $\mathrm{U}$ between the system and probe qubit, with

$$
\mathrm{U}=\mathrm{C}-\mathrm{NOT} \cdot \mathrm{C}-S(\theta) \cdot \mathrm{C}-\mathrm{NOT}
$$

as shown in Fig. 2(b) in the main text. Let X, Y, and Z be the Pauli matrices, $\mathbb{1}$ be the identity matrix, and $\{|0\rangle,|1\rangle\}$ be the eigenvector of $Z$ with eigenvalues $z=\{1,-1\}$. Then, C-NOT is the controlled-NOT gate which is defined in the form of

$$
\mathrm{C}-\mathrm{NOT}=|H\rangle\left\langle H\left|\otimes \mathbb{1}_{p}+\right| V\right\rangle\langle V| \otimes X
$$

and implemented by a PBS with the system qubit as the control and the probe qubit as the target. While

$$
\begin{aligned}
\mathrm{C}-S(\theta)= & \mathbb{1} \otimes\left|0_{p}\right\rangle\left\langle 0_{p}\right|+(\cos 2 \theta Z \\
& +\sin 2 \theta X) \otimes\left|1_{p}\right\rangle\left\langle 1_{p}\right|
\end{aligned}
$$


(a)

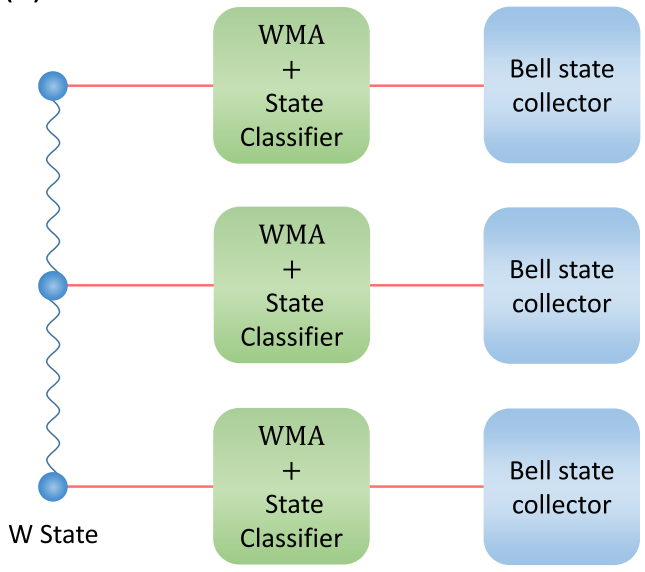

(b)

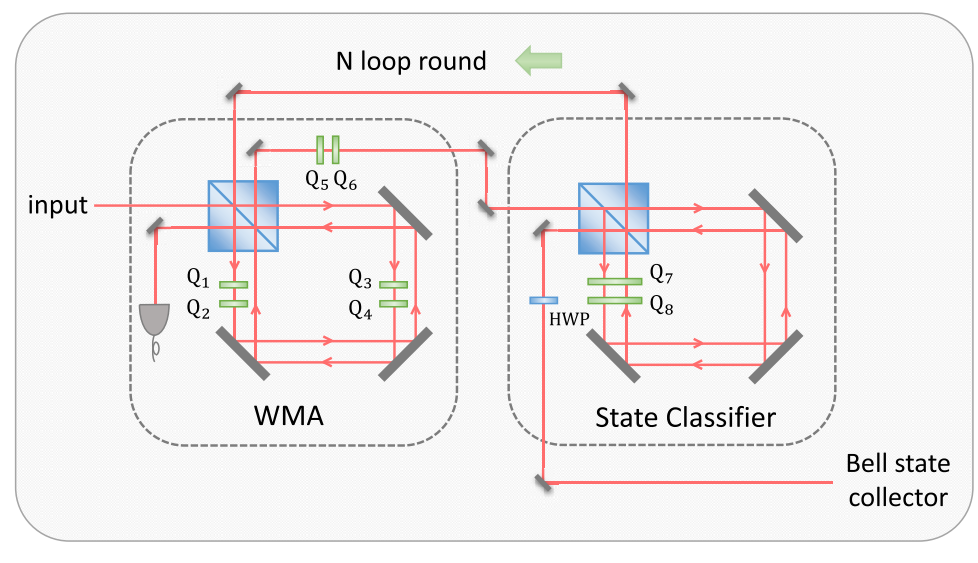

FIG. 6. Experimental setup of $N$-round distillation protocol.

is the controlled-rotation gate which is implemented by a halfwave plate (HWP) oriented at $\theta$ with the probe qubit as the control and system qubit as the target. Then, the two-qubit controlled-unitary gate $\mathrm{C}-\mathrm{U}$ is

$$
\begin{aligned}
\mathrm{U}= & \mathrm{C}-\mathrm{NOT} \cdot \mathrm{C}-S(\theta) \cdot \mathrm{C}-\mathrm{NOT} \\
= & (|H\rangle\langle H|-\cos 2 \theta| V\rangle\langle V|) \otimes\left|0_{p}\right\rangle\left\langle 0_{p}\right| \\
& +(|V\rangle\langle V|+\cos 2 \theta| H\rangle\langle H|) \otimes\left|1_{p}\right\rangle\left\langle 1_{p}\right| \\
& +\sin 2 \theta|H\rangle\left\langle V|\otimes| 1_{p}\right\rangle\left\langle 0_{p}\right| \\
& +\sin 2 \theta|V\rangle\left\langle H|\otimes| 0_{p}\right\rangle\left\langle 1_{p}\right| .
\end{aligned}
$$

In the experiment, the probe qubit is initialized as state $\left|0_{p}\right\rangle$, and measured in the $Z_{p}$ basis. Then, the measurement operators of the weak measurement on the system qubit is given by

$$
\begin{aligned}
& M_{+}=\left\langle 0_{p}|\mathrm{U}| 0_{p}\right\rangle=|H\rangle\langle H|-\cos 2 \theta| V\rangle\langle V|, \\
& M_{-}=\left\langle 1_{p}|\mathrm{U}| 0_{p}\right\rangle=\sin 2 \theta|H\rangle\langle V| .
\end{aligned}
$$

And the corresponding POVM elements are

$$
\begin{aligned}
& \mathcal{O}_{+}=M_{+}^{\dagger} M_{+}=\left(1-\frac{\sin ^{2} 2 \theta}{2}\right) \mathbb{1}+\frac{\sin ^{2} 2 \theta}{2} Z, \\
& \mathcal{O}_{-}=M_{-}^{\dagger} M_{-}=\frac{\sin ^{2} 2 \theta}{2} \mathbb{1}-\frac{\sin ^{2} 2 \theta}{2} Z .
\end{aligned}
$$

Defining $R=\sin ^{2} \theta$ as the measurement strength, we obtain the same form of POVM elements of Eq. (2) in the main text. Note that $\theta$ is the angle of the HWP in the Sagnac interferometer, and we can continuously change the measurement strength by adjusting the HWP angle $\theta$.

\section{APPENDIX D: ARBITRARY $N$-ROUND DISTILLATION PROTOCOL}

To achieve an arbitrary $N$-round distillation, we also designed an experimental setup based on Sagnac interferometers and high speed feedback operations. The setup for each one (Alice, Bob, or Charlie) could be divided into three parts: a WMA with continuously adjustable measurement strength similar to that we have implemented in the experiment, a state classifier, and a Bell state collector. In the following, we will give a detailed description for each part. We introduce the path degree of freedom of photons as the probe qubit with $\left|0_{p}\right\rangle\left(\left|1_{p}\right\rangle\right)$ representing the clockwise (counterclockwise) propagation states in the Sagnac interferometer.

\section{Weak measurement apparatus}

The WMA of the $N$-round distillation setup is also implemented by a Sagnac interferometer with some wave-plates. The only change is that at each path of the Sagnac interferometer and the output port of the WMA we introduce two quarter wave-plates (QWP) with high-speed control as shown in Fig. 6. With practical apparatus, the function of QWP with high-speed control can be implemented with the Pockels cells, which are often made by potassium dihydrogen phosphate crystal or other nonlinear crystals, and could be controlled via a high-speed variable voltage.

At the first round, the photon of the tripartite $W$ state shot into the Sagnac interferometer from the input port, and we set the angles of the six QWPs to be $\theta, \theta, 0^{\circ}, 90^{\circ}$, $0^{\circ}$, and $90^{\circ}$, where the optimal $\theta$ is determined by the total distillation rounds as shown in the main text. Then, the QWPs $\mathrm{Q}_{1}$ and $\mathrm{Q}_{2}$ have the same function as the half-wave plate (HWP) in our experiment and could also implement a controlled-rotation gate C-S $(\theta)=\mathbb{1} \otimes\left|0_{p}\right\rangle\left\langle 0_{p}\right|+(\cos 2 \theta Z+$ $\sin 2 \theta X) \otimes\left|1_{p}\right\rangle\left\langle 1_{p}\right|$ with the probe qubit as the control and system qubit as the target. The $\mathrm{Q}_{3}$ and $\mathrm{Q}_{4}\left(\mathrm{Q}_{5}\right.$ and $\left.\mathrm{Q}_{6}\right)$ implement an identity transform with the setting above. Then with the setting of angles of six QWPs above, the WMA of $N$-round distillation setup is same as the WMA that we have implemented in the experiment.

At the $n$th round $(2 \leqslant n \leqslant N)$, the photon of the tripartite $W$ state is guided into the WMA again from the other input port of the Sagnac interferometer after the classify process of the state classifier. In this case, we set the angles of six QWPs to be $45^{\circ}, 45^{\circ}, 45^{\circ}+\theta, 45^{\circ}+\theta, 45^{\circ}$, and $45^{\circ}$, where the optimal $\theta$ is determined by the total distillation rounds and which round of the distillation process as shown in the main text. Then, the QWPs $\mathrm{Q}_{1}$ and $\mathrm{Q}_{2}$ implement an C-NOT gate

$$
\mathrm{U}_{\mathrm{Q}_{1} \mathrm{Q}_{2}}=\mathbb{1} \otimes\left|0_{p}\right\rangle\left\langle 0_{p}|+X \otimes| 1_{p}\right\rangle\left\langle 1_{p}\right|
$$


with the probe qubit as the control and system qubit as the target. Similar, the QWPs $\mathrm{Q}_{5}$ and $\mathrm{Q}_{6}$ implement an C-NOT gate

$$
\mathrm{U}_{\mathrm{Q}_{5} \mathrm{Q}_{6}}=\mathbb{1} \otimes\left|0_{p}\right\rangle\left\langle 0_{p}|+X \otimes| 1_{p}\right\rangle\left\langle 1_{p}\right| .
$$

The QWPs $\mathrm{Q}_{3}$ and $\mathrm{Q}_{4}$ implement an controlled-rotation gate

$$
\begin{aligned}
\mathrm{U}_{\mathrm{Q}_{3} \mathrm{Q}_{4}}= & \left(\cos 2\left(\theta+45^{\circ}\right) Z+\sin 2\left(\theta+45^{\circ}\right) X\right) \otimes\left|0_{p}\right\rangle\left\langle 0_{p}\right| \\
& +\mathbb{1} \otimes\left|1_{p}\right\rangle\left\langle 1_{p}\right| .
\end{aligned}
$$

The PBS in the Sagnac interferometer could also be represented as a C-NOT gate

$$
\mathrm{U}_{\mathrm{PBS}}=|H\rangle\left\langle H\left|\otimes \mathbb{1}_{p}+\right| V\right\rangle\langle V| \otimes X .
$$

with the system qubit as the control and the probe qubit as the target. Then the Sagnac interferometer could be represented by a two-qubit unitary gate

$$
\begin{aligned}
\mathrm{U}= & \mathrm{U}_{\mathrm{PBS}} \cdot \mathrm{U}_{\mathrm{Q}_{1} \mathrm{Q}_{2}} \cdot \mathrm{U}_{\mathrm{Q}_{3} \mathrm{Q}_{4}} \cdot \mathrm{U}_{\mathrm{PBS}} \cdot \mathrm{U}_{\mathrm{Q}_{5} \mathrm{Q}_{6}} \\
= & (|H\rangle\langle H|+\cos 2 \theta| V\rangle\langle H|) \otimes\left|0_{p}\right\rangle\left\langle 1_{p}\right| \\
& +(|V\rangle\langle V|+\cos 2 \theta| H\rangle\langle H|) \otimes\left|1_{p}\right\rangle\left\langle 0_{p}\right| \\
& +\sin 2 \theta|V\rangle\left\langle H|\otimes| 0_{p}\right\rangle\left\langle 0_{p}\right| \\
& -\sin 2 \theta|H\rangle\left\langle H|\otimes| 1_{p}\right\rangle\left\langle 1_{p}\right| .
\end{aligned}
$$

Here, the probe qubit is initialized to state $\left|1_{p}\right\rangle$, and measured in the $Z_{p}$ basis. Then, the measurement operators of the weak measurement on the system qubit can be given by

$$
\begin{aligned}
& M_{+}=\left\langle 0_{p}|\mathrm{U}| 1_{p}\right\rangle=|H\rangle\langle H|+\cos 2 \theta| V\rangle\langle V|, \\
& M_{-}=\left\langle 1_{p}|\mathrm{U}| 1_{p}\right\rangle=-\sin 2 \theta|H\rangle\langle V| .
\end{aligned}
$$

And the corresponding POVM elements are

$$
\begin{aligned}
& \mathcal{O}_{+}=M_{+}^{\dagger} M_{+}=\left(1-\frac{\sin ^{2} 2 \theta}{2}\right) \mathbb{1}+\frac{\sin ^{2} 2 \theta}{2} Z, \\
& \mathcal{O}_{-}=M_{-}^{\dagger} M_{-}=\frac{\sin ^{2} 2 \theta}{2} \mathbb{1}-\frac{\sin ^{2} 2 \theta}{2} Z .
\end{aligned}
$$

It has the same form with Eq. (C6). Then the WMA could perform the same weak measurement on the system qubit as the first round distillation. The measurement strength is $R=$ $\sin ^{2} \theta$, which can be continuously adjusted by changing the QWP's angle $\theta$.

At the final round, the photon of the tripartite $W$ state is also guided into the WMA after all the rounds of random distillation. Then one (suppose Charlie) set the parameter $\theta=45^{\circ}$. In this case, the measurement strength should be 1 , and he performs a strong measurement on the photon of $W$ state. While the other two (Alice and Bob) set the parameter $\theta=0^{\circ}$. The measurement strength is 0 and they do no operations on the photons of $W$ state. After the final round, we use up the remaining $W$ sate and accomplish the distillation process.

\section{State classifier}

The state classifier, implemented by a Sagnac interferometer with QWPs, is used to choose whether to lead photon back into the previous WMA or go straight to the Bell state collector. Suppose an arbitrary state $|\psi\rangle \otimes\left|0_{p}\right\rangle=\alpha|H\rangle \otimes\left|0_{p}\right\rangle+$ $\beta|V\rangle \otimes\left|0_{p}\right\rangle$ is input to the state classifier. As shown above, the PBS in the Sagnac interferometer could be represented a C-NOT gate

$$
\mathrm{U}_{\mathrm{PBS}}=|H\rangle\left\langle H\left|\otimes \mathbb{1}_{p}+\right| V\right\rangle\langle V| \otimes X,
$$

with the system qubit as the control and the probe qubit as the target.

When we want the system state back into the previous WMA, the angles of QWPs $\mathrm{Q}_{7}$ and $\mathrm{Q}_{8}$ are set as $0^{\circ}$ and $90^{\circ}$, respectively. They implement an identity transform on the system qubit and the output state is

$$
\left|\Psi_{\text {out }}\right\rangle=\mathrm{U}_{\mathrm{PBS}} \cdot \mathrm{U}_{\mathrm{PBS}}|\psi\rangle \otimes\left|0_{p}\right\rangle=|\psi\rangle \otimes\left|0_{p}\right\rangle .
$$

Then, the photon come out of the Sagnac interferometer and is guided back into previous WMA without any change of the state encoded in polarization degree of freedom.

When want the system state to go straight to the Bell state collector, the angles of $\mathrm{QWPs} \mathrm{Q}_{7}$ and $\mathrm{Q}_{8}$ are both set as $45^{\circ}$. We also set a HWP at $45^{\circ}$ at the path towards the Bell sate collector which can be represented in the form of

$$
\mathrm{U}_{\mathrm{HWP}}=\mathbb{1} \otimes\left|0_{p}\right\rangle\left\langle 0_{p}|+X \otimes| 1_{p}\right\rangle\left\langle 1_{p}\right| .
$$

The output state could be calculated as

$$
\begin{aligned}
\left|\Psi_{\text {out }}\right\rangle & =\mathrm{U}_{\mathrm{HWP}} \cdot \mathrm{U}_{\mathrm{PBS}} \cdot \mathrm{U}_{\mathrm{PBS}}|\psi\rangle \otimes\left|0_{p}\right\rangle \\
& =|\psi\rangle \otimes\left|1_{p}\right\rangle .
\end{aligned}
$$

Then, the photon comes out from the other output port of the Sagnac interferometer and goes straight to the Bell state collector. The state encoded in the polarization degree of freedom is not changed.

\section{Bell state collector}

The Bell state collector is used to collect and save the EPR state distilled from the $W$ state. In the distillation process, Alice, Bob, and Charlie send the photons of a single copy tripartite $W$ state into the setup, respectively, to implement an $N$-round entanglement distillation. With high-precision time control, we adjust the angles of the six QWPs of WMA $\left(\mathrm{Q}_{1}\right.$, $\mathrm{Q}_{2}, \ldots, \mathrm{Q}_{6}$ ) for each round as discussed before. When no detector of the three WMA clicks, the angles of the two QWPs in the state classifier $\left(\mathrm{Q}_{7}, \mathrm{Q}_{8}\right)$ are respectively set to be $0^{\circ}$ and $90^{\circ}$, and the photons of the $W$ state run loops in the setup. When only one out of the three detectors clicks, the other two share an EPR pair. We rotate both the angles of $\mathrm{Q}_{7}$ and $\mathrm{Q}_{8}$ to $45^{\circ}$ with high-speed feedback operations and the EPR pair will be guided into the Bell state collector. When more than one detector of the three WMAs clicks, the distillation fails. If there is no detector of the three WMAs clicking after $N$-round distillation, we do the final round distillation by perform a strong measurement on Charlie's photon to make full use of the remainder $W$ state and end the distillation process. 
[1] M. Epping, H. Kampermann, C. macchiavello, and D. Bruß, New J. Phys. 19, 093012 (2017).

[2] D. Bouwmeester, J.-W. Pan, K. Mattle, M. Eibl, H. Weinfurter, and A. Zeilinger, Nature (London) 390, 575 (1997).

[3] X.-S. Ma, T. Herbst, T. Scheidl, D. Wang, S. Kropatschek, W. Naylor, B. Wittmann, A. Mech, J. Kofler, E. Anisimova et al., Nature (London) 489, 269 (2012).

[4] J.-G. Ren, P. Xu, H.-L. Yong, L. Zhang, S.-K. Liao, J. Yin, W.-Y. Liu, W.-Q. Cai, M. Yang, L. Li, K.-X. Yang, X. Han, Y.-Q. Yao, J. Li, H.-Y. Wu, S. Wan, L. Liu, D.-Q. Liu, Y.-W. Kuang, Z.-P. He, P. Shang, C. Guo, R.-H. Zheng, K. Tian, Z.-C. Zhu, N.-L. Liu, C.-Y. Lu, R. Shu, Y.-A. Chen, C.-Z. Peng, J.-Y. Wang, and J.-W. Pan, Nature (London) 549, 70 (2017).

[5] A. Acín, D. Bruß, M. Lewenstein, and A. Sanpera, Phys. Rev. Lett. 87, 040401 (2001).

[6] Y. Takeuchi, N. Imoto, and T. Tashima, Phys. Rev. A 97, 042341 (2018).

[7] S. Wehner, D. Elkouss, and R. Hanson, Science 362, eaam9288 (2018).

[8] B. Fortescue and H.-K. Lo, Phys. Rev. Lett. 98, 260501 (2007).

[9] E. D'Hondt and P. Panangaden, Quantum Inf. Comput. 6, 173 (2006).

[10] V. Scarani, S. Iblisdir, N. Gisin, and A. Acín, Rev. Mod. Phys. 77, 1225 (2005).
[11] G. Gour, Phys. Rev. A 72, 042318 (2005).

[12] B. Fortescue and H.-K. Lo, Phys. Rev. A 78, 012348 (2008).

[13] W. Cui, E. Chitambar, and H.-K. Lo, Phys. Rev. A 84, 052301 (2011).

[14] Y. Aharonov, D. Z. Albert, and L. Vaidman, Phys. Rev. Lett. 60, 1351 (1988).

[15] M. Eibl, N. Kiesel, M. Bourennane, C. Kurtsiefer, and H. Weinfurter, Phys. Rev. Lett. 92, 077901 (2004).

[16] D. F. V. James, P. G. Kwiat, W. J. Munro, and A. G. White, Phys. Rev. A 64, 052312 (2001).

[17] M. Horodecki, J. Oppenheim, and A. Winter, Nature (London) 436, 673 (2005).

[18] J. A. Smolin, F. Verstraete, and A. Winter, Phys. Rev. A 72, 052317 (2005).

[19] O. Gühne, C.-Y. Lu, W.-B. Gao, and J.-W. Pan, Phys. Rev. A 76, 030305(R) (2007).

[20] M. Bourennane, M. Eibl, C. Kurtsiefer, S. Gaertner, H. Weinfurter, O. Gühne, P. Hyllus, D. Bruß, M. Lewenstein, and A. Sanpera, Phys. Rev. Lett. 92, 087902 (2004).

[21] P. B. Dixon, D. J. Starling, A. N. Jordan, and J. C. Howell, Phys. Rev. Lett. 102, 173601 (2009).

[22] S. Wu, Sci. Rep. 3, 1193 (2013).

[23] F. Kaneda, S.-Y. Baek, M. Ozawa, and K. Edamatsu, Phys. Rev. Lett. 112, 020402 (2014). 